\title{
Diagnosis and Management of Congenital Perilymph Fistula causing Recurrent Meningitis: A Review
}

\author{
Vedantam Rupa ${ }^{1}$ \\ 1Department of Ear, Nose \& Throat and Audiovestibular \\ Services (E.N.T.), Christian Medical College, Vellore, \\ Tamil Nadu, India
}

Ann Otol Neurotol ISO 2018;1:40-42

\begin{abstract}
Address for correspondence Vedantam Rupa, DLO, MS, Department of Ear, Nose \& Throat and Audiovestibular Services (E.N.T.), Christian Medical College, Vellore 632004, Tamil Nadu, India (e-mail: rupavedantam@cmcvellore.ac.in).
\end{abstract}

\begin{abstract}
Keywords

- congenital perilymph fistula

- inner ear anomaly

- recurrent meningitis

Background Congenital perilymph fistula is a rare cause of recurrent meningitis and should be suspected in any child who has had more than one episode of meningitis.

Aim To define the entity of congenital perilymph fistula causing recurrent meningitis and review the literature on the subject.

Methods A search was made in the PubMed database for all articles using the MeSH search terms "congenital perilymph fistula" AND "recurrent meningitis" AND "congenital perilymph fistula" AND "meningitis."

Results A total of 48 articles were obtained, of which only the relevant articles were reviewed. Diagnosis of this condition is based on typical radiological features seen on high-resolution CT scan or thin slice MRI scan of the temporal bone. A variety of congenital inner anomalies with typical radiological features may be associated with this condition. Clinical features are not diagnostic and delayed diagnosis may occur due to a low index of suspicion. All children should undergo otomicroscopic and audiological assessment too, as radiology may rarely be noncontributory. Surgical closure of these fistulae using a tympanotomy approach is successful in most patients.

Conclusion Congenital perilymph fistula causing recurrent meningitis is a rare entity whose diagnosis is dependent on a high index of suspicion and appropriate radiological assessment. Surgical closure of the fistula prevents further episodes of meningitis.
\end{abstract}

\section{Introduction}

Congenital perilymph fistula is a rare cause of recurrent meningitis. ${ }^{1}$ The diagnosis should be suspected in any child who has had more than one episode of meningitis. A comprehensive and exhaustive battery of tests should be ordered, as the source of meningitis could be elusive. Because of the rarity of its occurrence, the literature is replete with isolated case reports ${ }^{2-4}$ and a small series of patients..$^{5-7}$

\section{Clinical Presentation}

The episodes of recurrent meningitis usually commence within the first decade of life in children suffering from congenital perilymph fistula. There is an almost equal distribution in males and females. Most children have unilateral sensorineural hearing loss. These patients may have symptoms of hearing loss and vertigo. However, such symptoms are rarely volunteered in a child. Many such children are found to have unilateral hearing loss only on audiologic testing, as the parents appear to be unaware of the presence of this problem.

Diagnosis of this condition is often delayed because of a low index of suspicion. Establishing a causal relationship between the inner ear and repeated episodes of meningitis involves detailed audiologic and radiologic investigations. In some instances, clinical and radiologic evaluation may be normal, necessitating exploratory tympanotomy. ${ }^{3}$

Bacterial meningitis is a potentially life-threatening illness, and recurrent meningitis increases the morbidity and mortality risk severalfold. By definition, recurrent bacterial meningitis refers to a second episode of meningitis occurring at least 3 weeks after treatment of the previous one or if an 
organism different from the previous one has been isolated at the time of the second episode. ${ }^{8}$ Evaluation of these patients should be comprehensive and exhaustive, particularly if there is no obvious focus of infection in the ear or nose.

\section{Delayed Diagnosis}

One of the characteristics of these children is delayed diagnosis. Some of the earlier reports in the literature ${ }^{4,9} \mathrm{de}-$ scribe patients with several episodes of meningitis before diagnosis. Gundersen and Haye ${ }^{9}$ describe a 60 -year-old patient who had experienced 26 episodes of meningitis before a diagnosis was established. The lack of computed tomographic (CT) and magnetic resonance imaging (MRI) scanning facilities in that era could have led to delayed diagnosis. However, there have been reports with delayed diagnosis in recent times too. ${ }^{7,10}$ Delayed diagnosis in some of the older cases and many of the recently encountered cases of congenital perilymph fistulae is largely due to lack of familiarity with the mode of presentation of this entity by otolaryngologists and nonotolaryngologists alike.

In children or adults with meningitis, it is the pediatrician, neurologist, or internist who first encounters the patient. After treatment of meningitis, appropriate referral to the otolaryngologist is crucial. If this important step is not performed, particularly in children with recurrent meningitis, the child has to bear the consequences. With each episode of meningitis, the hearing also worsens and most children have a unilateral profound hearing loss. Detailed history (particularly of earache prior to onset of meningitis), pure tone audiometry or brainstem evoked response audiometry (depending on the child's age), and radiologic imaging by the otolaryngologist then helps localize the site of leak.

Most children with congenital inner ear anomalies have severe to profound sensorineural hearing loss, either due to the inner ear anomaly itself or due to subsequent labyrinthitis or meningitis. In rare situations, hearing in these children is normal, and the only method of diagnosis is by imaging., ${ }^{3,7}$

\section{Embryology}

The otic placodes develop from the ectoderm at the third week of intrauterine life and invaginate to form otic vesicles. ${ }^{11}$ These subsequently develop to form the endolymphatic sac, cochlea, and vestibule by the fifth week. The membranous cochlea develops 1.5 turns at the end of 6 weeks and 2.5 turns by the end of the seventh week. The semicircular canals develop by the eighth week. The development of the inner ear is almost complete by the eighth week, so any insult during this period can produce several anomalies including congenital perilymph fistula. Some of the inner ear developmental anomalies that are associated with a congenital perilymph fistula ${ }^{12}$ are:

- Common Cavity Anomaly: In common cavity anomaly, there is developmental arrest at the fourth week of gestation, leading to the formation of a single cavity that represents the undifferentiated cochlea and vestibule ( - Fig. 1). This is often associated with oval or round window fistula.

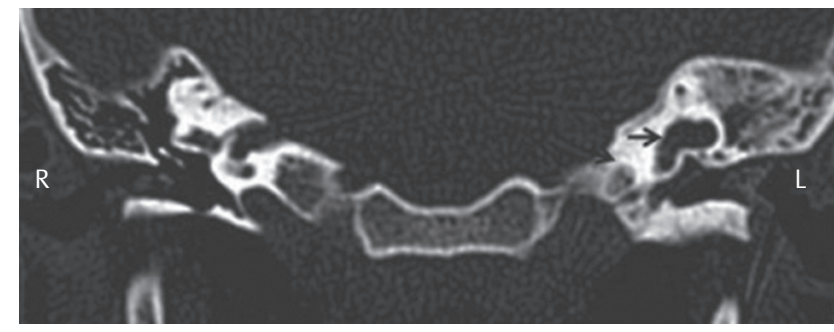

Fig. 1 High-resolution coronal CT scan showing common cavity defect with no definable partitions (arrow). CT, computed tomography; $\mathrm{L}$, left; R, right.

- Incomplete Partition Type 1: In incomplete partition type 1 , also known as cystic cochleovestibular malformation ( - Fig. 2), cochlea has no bony modiolus and results in an empty cystic cochlea and dilated cystic vestibule. The developmental arrest occurs at the fifth week of gestation.

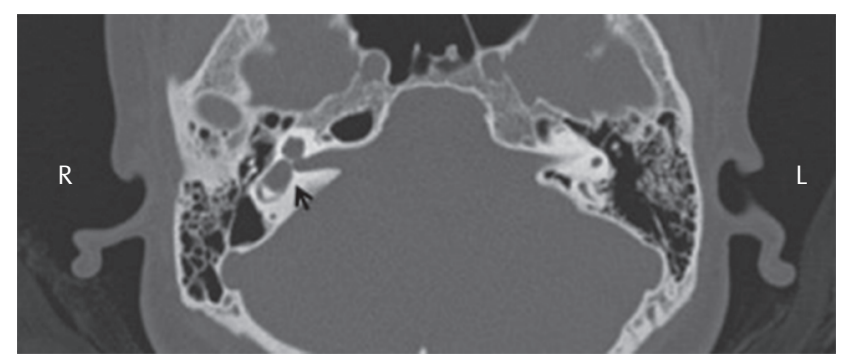

Fig. 2 High-resolution axial CT scan showing type 1 incomplete partition defect; the cochlea and vestibule are separately defined, but they are featureless; arrow shows dilated, featureless vestibules. CT, computed tomography; L, left; R, right.

- Incomplete Partition Type 2: Incomplete partition type 2 occurs when the apical and middle turn of cochlea are not developed resulting in a cystic apex. It also called Mondini's deformity. The cochlea consists of only 1.5 turns. The vestibule is normal, whereas the vestibular aqueduct is enlarged. The developmental arrest occurs at the seventh week of gestation. This is often associated with oval window fistula.

\section{Anatomical Abnormalities}

Several temporal bone and ossicular abnormalities occur in these patients. The basic abnormality is a fistulous communication between the inner ear and the intracranial compartment as well as another communication between the inner ear and middle ear. In some cases, as in Hyrtl's fissure, there is a direct communication between the subarachnoid space of the posterior cranial fossa and the middle ear.

The fistulous communication between the middle and inner ear that has been described in the literature includes oval window fistula,,${ }^{4,5,13}$ round window fistula, ${ }^{13}$ promontory defect, ${ }^{13}$ Hyrtl's fissure, ${ }^{13}$ roof of Eustachian tube,,$^{13}$ and anterior to semicircular canals. ${ }^{14}$ The oval and round windows are, in fact, the most common sites of fistulae.

The communication between the subarachnoid space and inner ear that has been described includes defects in the lateral aspect of the internal auditory canal or facial canal or a 
widely patent cochlear aqueduct. Rarely, a direct communication between the subarachnoid space of the posterior cranial fossa and the middle ear occurs, and this is known as Hyrtl's fissure. This opening lies close to the round window niche.

Other anomalies noted are malformed stapes, often with a single crus.

\section{Imaging}

The best imaging modalities to demonstrate these anatomical abnormalities are high-resolution CT scan of the temporal bone and thin slice MRI scanning of the temporal bone. ${ }^{15,16}$ Whereas CT is excellent to delineate bony defects, MRI is invaluable in demonstrating the fluid-filled communications and nerves. Some authors have found that three-dimensional (3D) CT scanning of the temporal bone could detect minute abnormalities that are not evident on two-dimensional (2D) CT scan. ${ }^{17}$ Inner ear anomalies such as Mondini's deformity and types 1 and 2 inner ear anomalies are evident on both CT and MR scanning. The communication between the cerebrospinal fluid (CSF) spaces and the inner ear at the lateral end of the internal auditory meatus or through a defect in the promontory is well seen on T2-weighted MR images. ${ }^{12}$

The presence of a normal CT and/or MRI scan in these patients is rare. ${ }^{7,14}$ In such situations, exploratory tympanotomy would be required to confirm diagnosis. ${ }^{3}$

\section{Surgical Management}

Surgical closure of these defects is the procedure of choice. In the early reports, several patients required multiple procedures to definitively close the fistula, and this was often evident only on long-term follow-up.

\section{Multiple Surgeries}

Surgical procedures for the management of small perilymph fistulae have usually involved simple closure of the defect after scarifying the edges ${ }^{5,9}$. However, in patients with recurrent meningitis due to a congenital perilymph fistula, more definitive procedures are required to prevent recurrence. ${ }^{5}$ Luntz et $\mathrm{al}^{5}$ describe a surgical failure rate of $38 \%$. Most patients failed when simple grafting was performed.

Vestibular obliteration is the definitive treatment of a congenital perilymph fistula. ${ }^{5}$ Some authors ${ }^{9}$ describe obliteration of the internal acoustic meatus only in recurrent fistulae. An argument against vestibular obliteration is the need to preserve residual hearing in the affected ear. As hearing loss in the affected ear is usually severe to profound in most children, this may not be a reason to avoid the procedure. Even in a child with normal hearing, vestibular obliteration is preferable if life-threatening meningitis is to be avoided. ${ }^{7}$ Strict bedrest for about 1 week in the immediate postoperative period is also recommended.

\section{Recurrence after Definitive Surgery}

Recurrence following an initial attempt or attempts at closure of fistula has been described by several authors. ${ }^{4,5}$ Luntz et al, ${ }^{5}$ reviewing 21 previously published cases of large oval window fistulae, found that primary surgery for closure of the fistula through a transaural approach succeeded only in 9.5\%. Overall, when closure was attempted through either a transaural or transcranial route, only $24 \%$ were successful after a single surgery. Surgical closure was attempted up to three to four times in up to $38 \%$ of cases. In most of the cases that failed, the primary surgery had been simple closure of the fistula with fascial graft. Up to $62 \%$ of patients who had undergone complete vestibular obliteration did not have a recurrence.

\section{Conclusion}

In conclusion, congenital perilymph fistula is an important cause of recurrent meningitis in children and adults requiring a high index of suspicion for diagnosis. Definitive evaluation includes high-resolution CT and MRI of the temporal bone. Negative imaging studies should be followed by exploratory tympanotomy. Vestibular obliteration rather than simple fistula closure is the treatment of choice.

\section{References}

1 Drummond DS, de Jong AL, Giannoni C, Sulek M, Friedman EM. Recurrent meningitis in the pediatric patient-the otolaryngologist's role. Int J Pediatr Otorhinolaryngol 1999;48(3):199-208

2 Herther C, Schindler RA. Mondini's dysplasia with recurrent meningitis. Laryngoscope 1985;95(6):655-658

3 Rupa V, Rajshekhar V, Weider DJ. Syndrome of recurrent meninigitis due to congenital perilymph fistula with two different clinical presentations. Int J Pediatr Otorhinolaryngol 2000;54(2-3):173-177

4 Clark JL, DeSanto LW, Facer GW. Congenital deafness and spontaneous CSF otorrhea. Arch Otolaryngol 1978;104(3):163-166

5 Luntz M, Frank I, Yurovitzki I, Berger G, Sadé J. Large perilymph fistulas. Am J Otol 1986;7(4):282-286

6 Althaus SR. Perilymph fistulas. Laryngoscope 1981;91(4): 538-562

7 Rupa V, Agarwal I, Rajshekhar V. Congenital perilymph fistula causing recurrent meningitis: lessons learnt from a single-institution case series. Otolaryngol Head Neck Surg 2014;150(2):285-291

8 Kline MW. Review of recurrent bacterial meningitis. Pediatr Infect Dis J 1989;8(9):630-634

9 Gundersen T, Haye R. Cerebrospinal otorrhea. Arch Otolaryngol 1970;91(1):19-23

10 Syal R, Tyagi I, Goyal A. Cerebrospinal fluid otorhinorrhea due to cochlear dysplasias. Int J Pediatr Otorhinolaryngol 2005;69(7):983-988

11 Jackler RK, Luxford WM, House WF. Congenital malformations of the inner ear: a classification based on embryogenesis. Laryngoscope 1987;97(3 Pt 2, Suppl 40):2-14

12 Sennaroglu L, Saatci I. A new classification for cochleovestibular malformations. Laryngoscope 2002;112(12):2230-2241

13 Parisier SC, Birken EA. Recurrent meningitis secondary to idiopathic oval window CSF leak. Laryngoscope 1976;86 (10): 1503-1515

14 Gacek RR, Leipzig B. Congenital cerebrospinal otorrhea. Ann Otol Rhinol Laryngol 1979;88(3 Pt 1):358-365

15 Johnson DB, Brennan P, Toland J, O’Dwyer AJ. Magnetic resonance imaging in the evaluation of cerebrospinal fluid fistulae. Clin Radiol 1996;51(12):837-841

16 Gupta V, Goyal M, Mishra NK, Sharma A, Gaikwad SB. Positional MRI: a technique for confirming the site of leakage in cerebrospinal fluid rhinorrhoea. Neuroradiology 1997;39(11):818-820

17 Isono M, Murata K, Aiba K, Miyashita H, Tanaka H, Ishikawa M. Minute findings of inner ear anomalies by three-dimensional CT scanning. Int J Pediatr Otorhinolaryngol 1997;42(1):41-53 